\title{
The Structure of Scientific Collaboration Networks in Scientometrics
}

\author{
HAIYAN HOU ${ }^{1,2}$ \\ HILDRUN KRETSCHMER ${ }^{1,3}$ \\ ZEYUAN LIU ${ }^{1,2}$
}

1 WISE LAB

Dalian University of Technology, Dalian, 116023, China

2 Institute of Science Studies and Management of Science and Technology

Dalian University of Technology, Dalian, 116023, China

3 Department of Library and Information Science

Humboldt-University of Berlin, Dorotheenstr. 26, D-10099 Berlin, Germany

\section{Abstract}

The structure of scientific collaboration networks in scientometrics was investigated at the level of individuals by using bibliographic data of all papers published in the international journal Scientometrics retrieved from the Science Citation Index (SCI) during 1978 to 2004. Combined analysis of social network analysis (SNA), cooccurrence analysis, cluster analysis and frequency analysis of words was explored to reveal: (1) The microstructure of the collaboration network on scientists' aspects of scientometrics; (2) The major collaborative fields of the collaborative sub-networks; (3) The collaborative center of the collaboration network in scientometrics.

\section{Introduction}

Since the pioneering work of Price [1] and Beaver \& Rosen [2][3][4], a large number of scholars have stressed different forms and roles of scientific collaboration in different scientific fields. The investigation of these researches can be made by analysis at micro level (individuals), meso level (institutions), or macro level (countries) [5][6]. In the field of scientometrics and informetrics many studies on international cooperation devoted into collaboration patterns and relationships between organizations and institutions instead of constructing micro collaboration networks on scientists aspects. [6][7] Schubert [8] and Dutt etc. [9] presented international collaboration characteristics in the scientometrics community itself, focusing on country aspects at macro level.

Using social network analysis, Newman [7] began to construct the actual collaboration networks between scientists in the field of physics, biomedical research, and computer science. However, Newman [10] pointed out that bibliometric analysis examining networks of individuals is not so easy to find. Kretschmer [6] appealed to devote more efforts to investigations at micro level in the future because the knowledge at meso and macro level does not yet adequately reflect the trends in cooperation between individuals. In the present study, we attempt to construct actual collaboration networks between scientometricians at micro level to reveal the structure of collaboration network in the field of scientometrics. 


\section{Data and Methods}

The study is based on bibliographic data retrieved from the Web of Science. The data contains all types of documents published in Scientometrics during 1978 to 2004. The data of each document includes author names, title, abstract, date, document type, addresses, and cited references. Author names were standardized because some authors may report their names differently in different papers. We identified each author by his or her surname and first initial only [7]. The retrieval was finally updated on May 25, 2005.

In this study we have adapted an integrated procedure of social network analysis (SNA), cooccurrence analysis, cluster analysis and frequency analysis of title words. Bibexcel is designed as a tool for manipulating bibliographic data, which is a free online-software published by Persson ${ }^{1}$. In the present study, Bibexcel is used to do co-occurrence analysis and cluster analysis. Social network analysis (SNA) was proved to be successful in studies of collaboration in bibliographic co-authorship networks and studies on networks visible on the Web [11] [12]. Following the methods of Otte \& Rousseau [11], White [13] and Kretschmer \& Aguillo [12], SNA was applied to display the microstructure of collaboration networks in scientometrics with Pajek ${ }^{2}$.

Moreover, we used frequency analysis of title words to display the main collaborative field of different sub-networks. The software for frequency analysis is demo version of Wordsmith Tools published by Oxford University Press and available online ${ }^{3}$.

\section{Results and discussion}

\subsection{Basic results}

There were 1927 documents published in Scientometrics during 1978 to 2004 (see Table 1). The total number of authors is 1630 . The total number of co-authors (the number of co-authors of a paper is equal to the number of collaborators of the paper) is 3340. Mean papers per author and mean authors per paper are 1.18 and 1.73 respectively. From Table 1, we found that the pattern of co-authorship was still dominated by single authored papers as the conclusion drawn by Dutt etc. [9]. Taking no account of single authored papers, the number of two authored papers is the most, accounting for $59.66 \%$ of the 875 co-authorship papers. The number of three authored papers accounts for $26.63 \%$. While the number of multi-authored papers (the number of co-authors is more than 3 ) accounts for $13.71 \%$ only, which indicates that team size in scientometrics is not large.

Table 1: Summary of basic results of the whole dataset.

\footnotetext{
${ }^{1}$ Bibexcel [CP]. http://www.se/inforsk/Bibexcel/

${ }^{2}$ Pajek [CP]. http://vlado.fmf.uni-lj.si/pub/networks/pajek/default.htm.

${ }^{3}$ Wordsmith Tools [CP]. http://www.lexically.net/wordsmith/index.html.
} 


\begin{tabular}{lc}
\hline & Basic results \\
\hline Total papers & 1927 \\
Single authored papers & 1052 \\
Co-authorship papers & 875 \\
$\quad$ Two authored papers & 522 \\
Three authored papers & 233 \\
Multi-authored papers & 120 \\
Total number of authors & 1630 \\
Total number of co-authors & 3340 \\
Mean paper per author & 1.18 \\
Mean authors per paper & 1.73 \\
Authors published 3 or more papers & 234 \\
Authors published co-authorship papers & 163 \\
\hline
\end{tabular}

\subsection{Microstructure of the collaboration network}

Bibexcel was used to do co-occurrence analysis to extract author names to list the collaborative pairs, and then do cluster analysis to identify the sub-networks, which represented different collaborative communities in the whole network. We used Pajek to perform social network analysis to construct the map of the collaboration network. In order to show the main structure of the network, each author must published 3 papers or more to be included in this integrated analysis. This threshold resulted in a total of 234 prolific authors publishing 3 or more papers during 1978 to 2004, among them there are 163 authors published co-authorship papers, accounting for $69.66 \%$ of the prolific authors.

These 163 authors formed an undirected co-authorship map visualizing the structure of collaboration network in the field of scientometrics (see Fig 1). The Kamada-Kuwai spring embedder in Pajek placed 163 nodes freely from a circular starting position [13]. We repositioned some authors slightly to prevent overlapping labels and varied the sizes of the authors by reweighting them by their degree centrality measure, which made the size of the authors proportional to their number of coworkers [13]. We resized the lines linking collaboration pairs with the number of co-authorship papers between the two collaborators.

Based on cluster analysis embedded in Bibexcel, we gained 22 clusters circled by solid lines (see Fig 1). We identified these clusters as sub-networks in the field of scientometrics. We numbered the sub-networks according to the size. The largest sub-network is number 1 that has 15 collaborators, and the second largest one is number 2, which has 14 collaborators, and so on. We noticed that there was totally 15 sub-networks connected with each other composing the largest central component, which had 96 numbers accounting for $58.90 \%$ of the prolific authors published co-authorship papers. Other 
authors formed one component of seven authors, one component of six authors, one component of four authors, four components of three authors, and 19 components of two authors.

Fig. 1: The microstructure of the collaboration network in the field of scientometrics, 1978-2004.

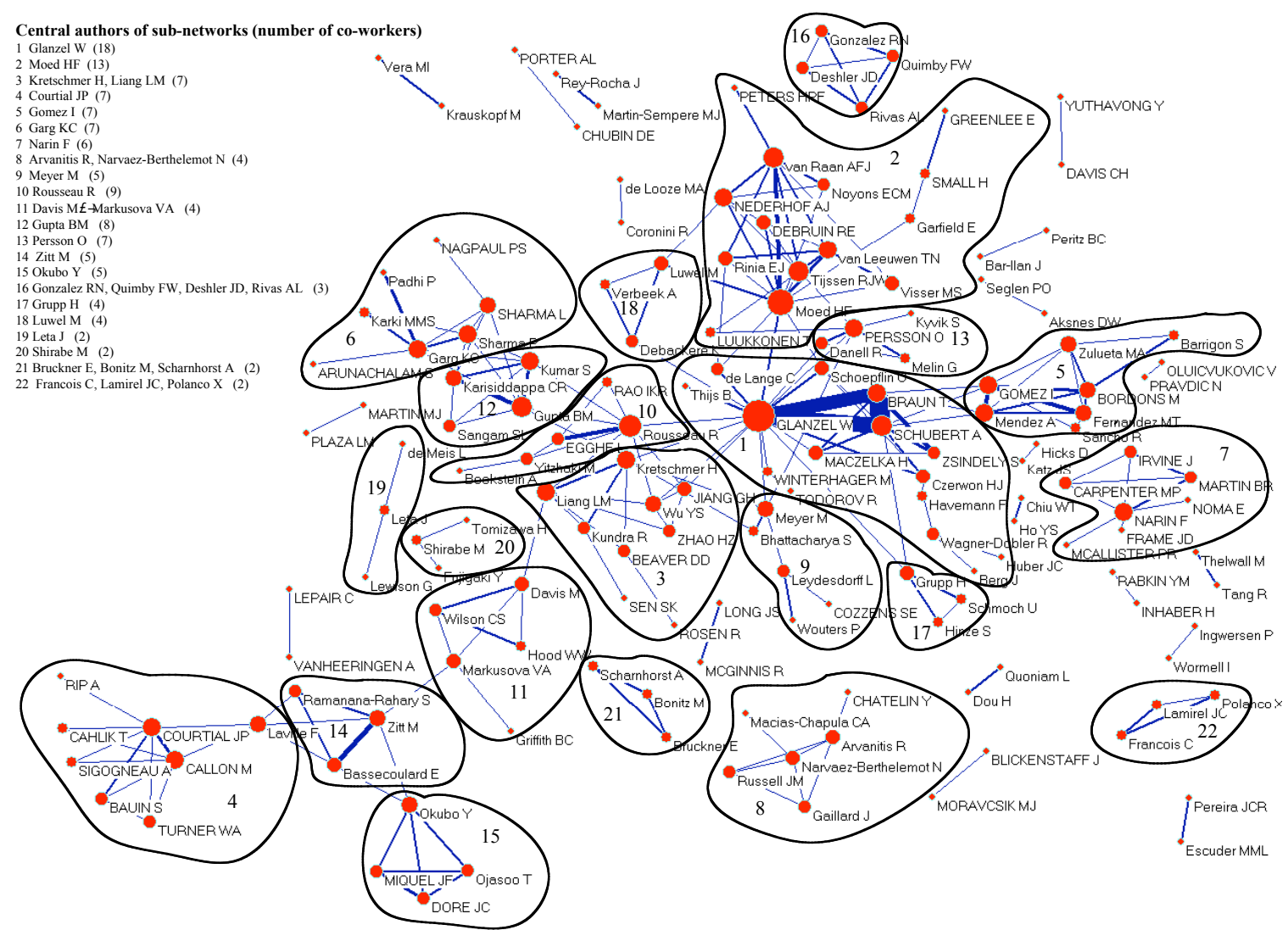

\section{Density and Centrality}

Density is an indicator for the general level of connectedness of the graph. According to Otte and Rousseau [11], the density of the network is defined by the number of links divided by the number of vertices in a complete graph with the same number of nodes. In the present study, there are totally 401 links in the network, so the density of the network is 0.03 , which indicates that the collaborative network in the field of scientometrics is very loose.

There are three centrality measures available in Pajek, degree, closeness and betweenness. In the collaborative network, degree centrality is equal to the number of co-workers or collaborators that an author has. So an author who has high degree centrality must has collaborated with many other authors, which means the author is a central collaborator of the whole network. In the present study, Glanzel who has 18 co-workers is the central author of the whole network.

According to Otte and Rousseau [11], closeness centrality of a node is equal to the total distance (in the graph) of this node from all other nodes. Glanzel has got the highest closeness in the whole network again. Betweenness centrality is the number of shortest paths that pass through a given node. Glanzel still has the highest betweenness, which indicates that he is the middleman connecting different groups and controlling the flow of information between most others [7] [11] [12]. We normalized the centrality by dividing the score by the maximum possible value. The three normed centralities of the top-ranked authors are listed as a percentage in Table 2 [13].

Table 2: Top ranks on normed centrality measures in collaboration networks in scientometrics. 


\begin{tabular}{cccccc}
\hline Degree & Score (\%) & Betweenness & Score (\%) & Closeness & Score (\%) \\
\hline Glanzel W & 11.11 & Glanzel W & 21.12 & Glanzel W & 19.36 \\
Moed HF & 8.02 & Liang LM & 11.87 & Rousseau R & 18.40 \\
Rousseau R & 5.56 & Davis M & 11.14 & $\begin{array}{c}\text { Kretschmer } \\
\text { H }\end{array}$ & 18.34 \\
Gupta BM & 4.94 & Markusova VA & 9.81 & Wu YS & 17.59 \\
Schubert A & 4.94 & Rousseau R & 9.17 & Meyer M & 16.75
\end{tabular}

In terms of the whole network, the degree centrality of the network is $9.44 \%$, which indicates that many authors do not collaborate at all. The network betweenness centrality is $20.38 \%$. The closeness centrality cannot be computed with Pajek since the network is not strongly connected.

Table 3: Correlations between output and centrality measures.

\begin{tabular}{c|cccc}
\hline Pearson Correlation & Output & Degree & Betweenness & Closeness \\
\hline Output & 1 & .648 & .473 & .338 \\
Degree & .648 & 1 & .685 & .461 \\
Betweenness & .473 & .685 & 1 & .406 \\
Closeness & .338 & .461 & .406 & 1 \\
\hline
\end{tabular}

With respect to sub-networks, there are also authors who have higher degree centrality than other authors in the same sub-network, so we identify such authors as the central authors of the subnetworks (see Fig 1). Five sub-networks have two or more central authors respectively who have the same score of degree centrality and the same number of co-workers. So there are 32 central authors in the whole network.

We found a positive and significant correlation between output of authors and the centrality measures $(\mathrm{r}=0.648,0.437,0.338$ respectively at the 0.01 level, see Table 3$)$ after investigating the correlations between output and the three centralities of the 125 authors in the 22 sub-networks, which indicated that most of the prolific authors are also active in collaboration network in the field of scientometrics.

\subsection{Collaborative fields of different sub-networks}

We counted the frequency of title words of the co-authorship papers published by the collaborators within each sub-network and listed the result in Fig 2. Terms with a frequency of one were excluded in order to exclude isolate aspects of collaborative field [14].

The biggest sub-network mainly concentrates on publication output and citation impact and other scientific indicators with scientometric analysis. The collaborative fields of the second largest subnetwork are similar to sub-network 1, but they use more bibliometric analysis than scientometric methods.

From Fig 2, we found some sub-networks having very clear and unique collaborative fields. Many authors of sub-network 3 are COLLNET members, and the two central authors Kretschmer and Liang 
together with Kundra are the very founders of COLLNET, which is a global interdisciplinary research network concentrating on the study of scientific collaboration.

From the frequency list of title words, we can clearly find that the main collaborative field of subnetwork 3 is scientific collaboration. In addition, because most of the authors are come from China and India, both "Chinese" and "Indian" occurred twice, revealing that the regional feature is also clear in this sub-network.

Sub-network 13 is another sub-network concentrating on scientific collaboration, which is led by Persson who collaborated with the other two colleagues to perform scientometric research in the Nordic countries, especially Sweden. Gupta and the other three Indian scientists modeled the scientific productivity and collaboration patterns in the area of theoretical population genetics in sub-network 12. Sub-network 5 took Spanish pharmacy and pharmacology as their main subjects as well as scientific collaboration. It is interesting that these four sub-networks dealing with scientific collaboration didn't collaborate with each other except sub-network 3 and 12 collaborating on collaboration patterns in theoretical population genetics via one co-authorship paper.

The most frequently occurred title word of sub-network 4 is co-word, which indicates the main collaborative field of Courtial and his collaborators is co-word analysis. Mapping of science is another method they used to study scientific research. As the central author in sub-network 7, Narin and others devoted themselves to bibliometric studies of science policy and scientific performance. Theory on "own-language preference", a new measure of relative language self-citation, is one of the favorite topics of Rousseau and others in sub-network 10. Davis and Markusova led the sub-network 11 to perform informetric studies on scientific literature, especially in Russia.

Fig. 2: Collaborative fields of different sub-networks. 


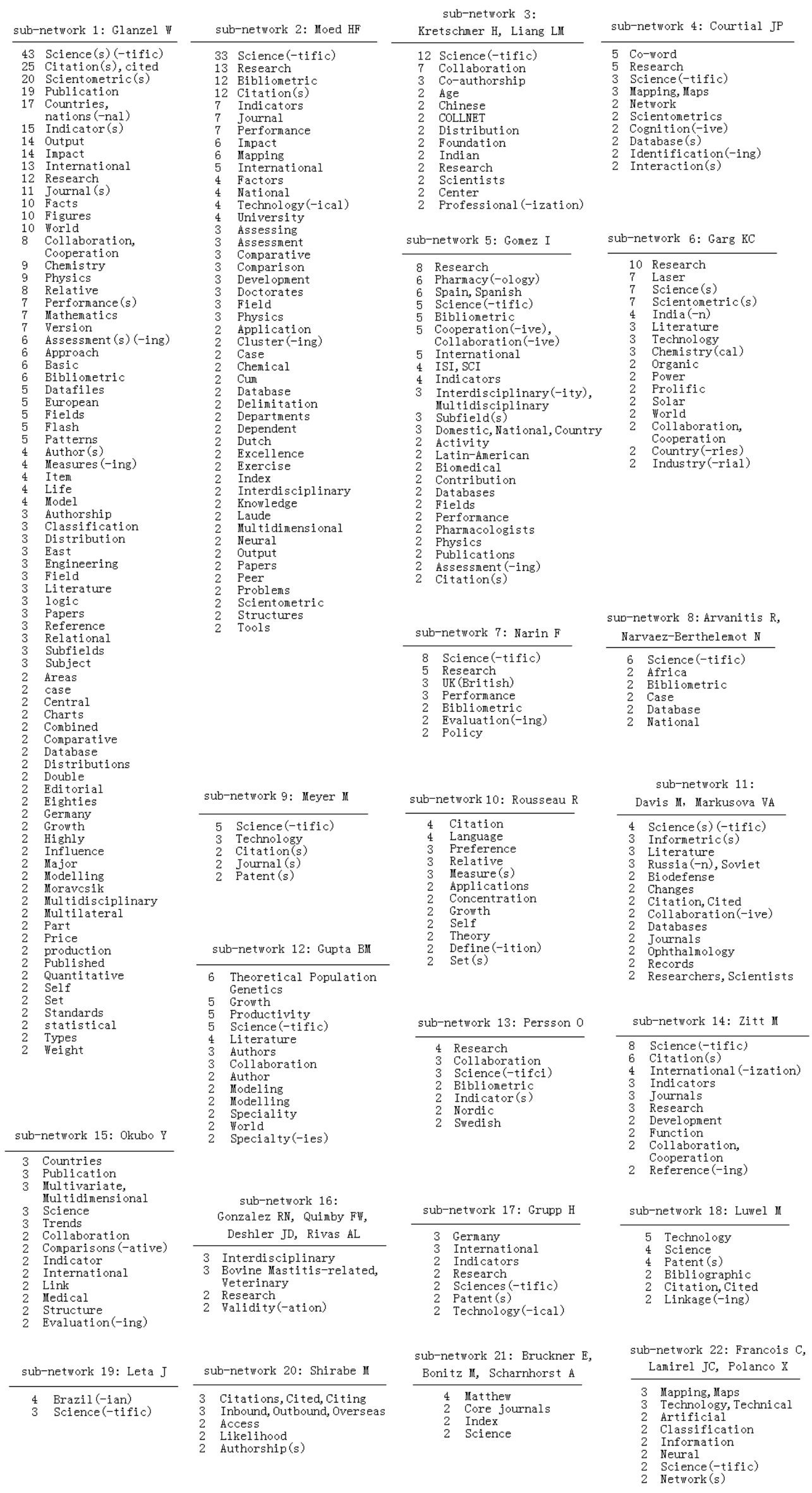


Four sub-networks focused on technology and science as their main collaborative topic, whereas, they never collaborated with each other on this topic at all. Scientometric study of laser science and technology in India is the most favorite collaborative field of sub-network 6 leading by Garg. Meyer and his collaborators in sub-network 9 characterized the intellectual spaces between science and technology. Science-technology interactions and linkage are the main topic of sub-network 18 leading by Luwel. Meanwhile, Francois, Lamirel and Polanco collaborated to perform mapping of science and technology based on artificial neural networks in sub-network 22 .

\subsection{Collaborative center}

In the whole collaboration network, sub-network 1 is the most influential sub-network, which collaborates with eight other sub-networks (see Fig 3). Sub-network 3 collaborates with 4 subnetworks. Sub-network 9, 10, 12, 13 and 14 collaborate with 3 other sub-networks respectively. There are two sub-networks have two collaborative sub-networks. And five sub-networks just link with one other sub-network.

We listed the top five collaboration pairs of the whole network (see Table 4). All of the top three pairs belong to sub-network 1 and the three authors involved are working in the same Hungarian institute. In Fig 1, we can clearly notice that the lines linking Glanzel, Braun and Schubert are significantly thick, forming the closest linking and the most active collaborative triple of the whole network. Linking with many sub-networks and containing the central author and the most active collaborative triple, sub-network 1 is identified to be the core collaborative center of the whole collaboration network in the field of scientometrics.

Table 4: Top five collaboration pairs.

\begin{tabular}{ccc}
\hline Collaboration pairs & Co-authorship papers & Number of sub-network \\
\hline Braun T/ Schubert A & 40 & 1 \\
Glanzel W/ Schubert A & 32 & 1 \\
Braun T / Glanzel W & 27 & 1 \\
Bassecoulard E/ Zitt M & 10 & 14 \\
Gupta BM/ Karisiddappa CR & 9 & 12
\end{tabular}

From Fig 3, we noticed that sub-network 1 and 2 collaborated through three co-authorship papers (two of them are editorial materials) concentrating on journal impact measures. The two co-authorship papers between sub-network 1 and 13 were mainly about scientific collaboration and science indicators. Sub-network 1 and 17 mainly collaborated on the scientometrics weight of 50 nations in 27 scientific areas. The two co-authorship papers between sub-network 1 and 3 are all prefaces. In terms of the number of co-authorship papers between sub-network 1 and the others, we can make the conclusion that although sub-network 1 links with many other sub-networks, it has not yet become a strong collaborative center of the whole network.

Fig. 3: Collaborative center of the collaboration network. 


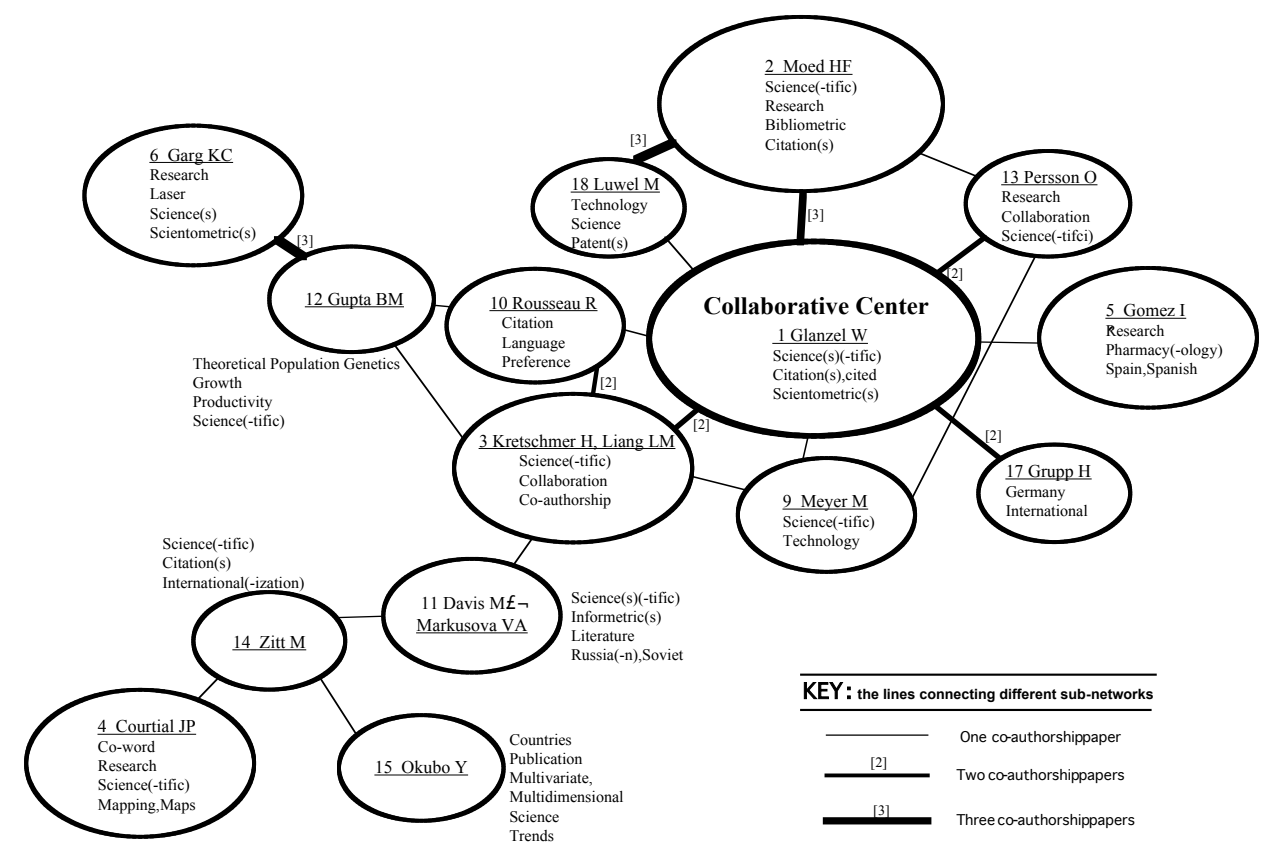

\section{Conclusions}

By using integrated analysis of social network analysis (SNA), co-occurrence analysis, cluster analysis and frequency analysis of words, we have constructed and visualized the microstructure of the scientific collaboration network in scientometrics. We found that there were 163 authors in the whole network and among them there were 96 authors connected with each other that composed a very large central component.

We also noted that the density, the degree centrality, and the betweenness centrality of the whole network were all very low, which indicated that the network was not strongly connected and the collaborative network in the field of scientometrics was very loose.

We have also investigated the centrality measures on each author. The result shows that Glanzel is the central author of the whole network in terms of the highest degree, betweenness and closeness centralities, which indicates that he is the most influential person in the network. With respect to subnetworks, we have identified the 32 central authors of sub-networks. Moreover, through correlation analysis, we found a positive and significant correlation between output of authors and the centrality measures, which revealed that most of the prolific authors were also active in collaboration network in the field of scientometrics.

We have presented the main collaborative field of different sub-networks in scientometrics and found that the two biggest sub-networks have the similar collaborative topic with slightly methodological difference. In addition, we found an interesting phenomenon that four sub-networks dealing with scientific collaboration didn't collaborate with each other except sub-network 3 and 12 . Moreover, four sub-networks studying technology and science never collaborated with each other at all.

We noticed that sub-network 1 was the core collaborative center of the whole collaboration network in the field of scientometrics, which collaborated with eight other sub-networks and included the central author and the most active collaborative triple. Nevertheless, in terms of the small number of co-authorship papers between sub-network 1 and the others, we have made the conclusion that the linkage between sub-network 1 and others was weak and it has not yet become a strong collaborative center of the whole network.

The investigation in the present study still represents a small part of the whole study. "Networks change over time, both because people enter and leave the professions they represent and because 
practices of scientific collaboration and publishing change. [7]" In our subsequent study, we will examine the dynamics of the collaboration network in the field of scientometrics.

\section{References}

1. D. J. de Solla. Price, Little science, big science, Columbia University Press, New York, 1963.

2. D. deB. Beaver and R. Rosen, Studies in scientific collaboration. 1. Scientometrics, 1: 6584, Sage, 1978.

3. D. deB. Beaver and R. Rosen, Studies in scientific collaboration. 2. Scientometrics, 2: 231245, Sage, 1979.

4. D. deB. Beaver and R. Rosen, studies in scientific collaboration. 3. Scientometrics, 3: 133149, Sage, 1979.

5. W. Glanzel, Coauthorship patterns and trends in the sciences (1980-1998): A bibliometric study with implications for database indexing and search strategies, Library Trends, 50:461473, Johns Hopkins University Press, 2002.

6. H. Kretschmer, Author productivity and geodesic distance in bibliographic co-authorship networks, and visibility on the Web, Scientometrics, 60:112, Kluwer, 2004.

7. M. Newman, Who is the best connected scientist? A study of scientific coauthorship networks, http://ideas.repec.org/p/wop/snfiwp/01-12-064.html, 2001.

8. A. Schubert, The web of scientometrics: A statistical overview of the frist 50 volumes of the journal Scientometrics, Scientometrics, 53:320, Kluwer, 2002.

9. B. Dutt, K. C. Garg and A. Bali, Scientometircs of the international journal Scientometircs, Scientometrics, 56:8193, Kluwer, 2003.

10. M. Newman, The structure of scientific collaboration networks, PNAS, 98:404409, the National Academy of Science, 2001.

11. E. Otte and R. Rousseau, Social network analysis: a powerful strategy, also for the information sciences, Journal of Information Science, 28:441453, Sage, 2002.

12. H. Kretschmer and I. Aguillo, Visibility of collaboration on the Web. Scientometrics, 61:405426, Kluwer, 2004.

13. H. D. White, Pathfinder networks and author cocitation analysis: A remapping of paradigmatic information scientists. Journal of the American Society for Information Science and Technology, 54: 423434, John Willey and Sons, 2003.

14. R. R. Braam, H. F. Moed and A. F. J. VanRaan, Mapping of science by combined co-citation and word analysis._. Structural aspects, JASIS, 42: 233251, John Willey and Sons, 1991. 